\title{
Combining Carcinoembryonic Antigen and Platelet to Lymphocyte Ratio to Predict Brain Metastasis of Resected Lung Adenocarcinoma Patients
}

\author{
Wei Wang, ${ }^{1,2}$ Chao Bian, ${ }^{3}$ Di Xia, ${ }^{3}$ Jin-Xi He, ${ }^{4}$ Ping Hai, ${ }^{1,2}$ \\ Ren Zhao, ${ }^{1,2}$ and Yan-Yang Wang ${ }^{1,2}$ \\ ${ }^{1}$ Department of Radiation Oncology, General Hospital of Ningxia Medical University, Yinchuan, Ningxia 750004, China \\ ${ }^{2}$ Cancer Institute, Ningxia Medical University, Yinchuan, Ningxia 750004, China \\ ${ }^{3}$ Graduate School, Ningxia Medical University, Yinchuan, Ningxia 750004, China \\ ${ }^{4}$ Department of Thoracic Surgery, General Hospital of Ningxia Medical University, Yinchuan, Ningxia 750004, China
}

Correspondence should be addressed to Yan-Yang Wang; fdwyy1981@hotmail.com

Received 20 February 2017; Revised 24 April 2017; Accepted 10 May 2017; Published 31 May 2017

Academic Editor: Noriyoshi Sawabata

Copyright (C) 2017 Wei Wang et al. This is an open access article distributed under the Creative Commons Attribution License, which permits unrestricted use, distribution, and reproduction in any medium, provided the original work is properly cited.

\begin{abstract}
We aimed to evaluate the role of pretreatment carcinoembryonic antigen (CEA) and platelet to lymphocyte ratio (PLR) in predicting brain metastasis after radical surgery for lung adenocarcinoma patients. The records of 103 patients with completely resected lung adenocarcinoma between 2013 and 2014 were reviewed. Clinicopathologic characteristics of these patients were assessed in the Cox proportional hazards regression model. Brain metastasis occurred in 12 patients (11.6\%). On univariate analysis, N2 stage $(P$ $=0.013)$, stage III $(P=0.016)$, increased CEA level $(P=0.006)$, and higher PLR value $(P=0.020)$ before treatment were associated with an increased risk of developing brain metastasis. In multivariate model analysis, CEA above $5.2 \mathrm{ng} / \mathrm{mL}(P=0.014)$ and PLR $\geq$ $120(P=0.036)$ remained as the risk factors for brain metastasis. The combination of CEA and PLR was superior to CEA or PLR alone in predicting brain metastasis according to the receiver operating characteristic (ROC) curve analysis (area under ROC curve, AUC 0.872 versus 0.784 versus 0.704 ). Pretreatment CEA and PLR are independent and significant risk factors for occurrence of brain metastasis in resected lung adenocarcinoma patients. Combining these two factors may improve the predictability of brain metastasis.
\end{abstract}

\section{Introduction}

Lung cancer is the first cause of cancer death in the world, and adenocarcinoma is the most diagnosed histological subtype $[1,2]$. Although surgical resection offers the best curative option for early stage lung adenocarcinoma, recurrence after surgery is still a critical problem, especially for brain metastasis, which is the major contribution to cancer death $[3,4]$. In order to further improve the survival of early stage lung adenocarcinoma patients, prevention of the occurrence of brain metastasis is one of the treatment options. It is wellknown that prophylactic cranial irradiation (PCI) has been used in the treatment of small cell lung cancer patients, which can reduce the frequency of brain metastasis and prolong the survival $[5,6]$. However, the value of this approach in the management of non-small cell lung cancer (NSCLC) patients is still in doubt [7]. Hence, defining the predictive factors for brain metastasis development and identifying high risk lung adenocarcinoma patients who will benefit from PCI is meaningful [8-10].

Carcinoembryonic antigen (CEA) is an oncofetal protein attached to epithelial-cell apical membrane via its c-terminal glycosylphosphatidylinositol anchor, a member of the immunoglobulin superfamily of cell adhesion molecules (IgCAM) [11]. High serum CEA levels have been associated with brain metastasis development and poor prognosis in patients with advanced NSCLC [12-15]. However, as we know, the relationship between baseline serum CEA levels and the brain metastasis development in resected lung adenocarcinoma patients is still not clear. 
Inflammation is increasingly recognized as being closely associated with cancer initiation and development. Inflammation can enhance tumor growth, invasion, angiogenesis, and, eventually, metastasis [16, 17]. Therefore, markers of inflammation may provide useful information for cancer diagnosis and management. The platelet to lymphocyte ratio (PLR), defined as the absolute platelet count divided by the absolute lymphocyte count, is a representative index of systemic inflammation. Its prognostic value has been studied in many types of cancers, including breast cancer, ovarian cancer, pancreatic cancer, and colorectal cancer [1821]. Recent studies suggest a potential prognostic role of PLR in lung cancer patients [22-27]. However, to our knowledge, no research has evaluated the role of PLR in predicting brain metastasis development for lung adenocarcinoma patients.

In this study, we reviewed the patients with completely resected lung adenocarcinoma and aimed to identify the predictive role of CEA and PLR and the combination analysis of these two factors in metastasis to brain of the curatively resected lung adenocarcinoma patients.

\section{Methods}

2.1. Patient Population and Clinical Data Collection. The study included 103 patients with pathologically confirmed lung adenocarcinoma who had received complete resection at General Hospital of Ningxia Medical University from 2013 to 2014. Clinicopathologic information of these patients, including age, sex, smoking history, tumor location, histological grade, tumor size, lymph node metastasis, TNM stage, and postoperative treatment modalities, was obtained from electronic medical records. TNM stage was classified according to the UICC/AJCC 7th TNM staging system, published in 2009 [28]. The pretreatment CEA and hemoglobin level were also included in the analysis. The PLR was defined as the absolute platelet count divided by the absolute lymphocyte count. Patients who were previously diagnosed with cancer other than nonmelanomatous skin cancer and who did not undergo brain computed tomography (CT) scans or magnetic resonance imaging (MRI) as part of their preoperative staging procedure were excluded from the study. And also, patients with hematologic, autoimmune, or infectious diseases or who received preoperative anticancer therapy were excluded. This study was approved by the ethics committee of General Hospital of Ningxia Medical University (number 2016-198).

2.2. Treatment and Follow-Up. All of the patients received complete pulmonary resection and systematic node dissection of the ipsilateral hilar and mediastinal lymph nodes. The patients with stage IB or higher lung cancer were given postoperative adjuvant therapy according to the National Comprehensive Cancer Network (NCCN) guidelines.

The follow-up was defined from the time of pulmonary resection. Physical examination, complete blood test, enhanced CT for chest, and ultrasound examination for abdomen were performed every 6 months for 2 years.
Contrast enhanced CT or MRI of the brain was performed if brain metastasis was suspected or yearly. Disease progression and failure sites were determined by radiologic examination, histologic examination, or both. The median follow-up time of the whole study population was 30 months (range, 4-42 months).

2.3. Statistical Analysis. All continuous variables were dichotomized to categorical variables basis on the median values of the sample. Potential risk factors of developing brain metastasis were evaluated by univariable and multivariable Cox proportional hazard model. The area under the curve (AUC) was used to assess the predictive value of each risk factor. Brain metastasis free survival time was defined as the period from date of the pulmonary resection to brain metastasis or the last follow-up. The brain metastasis free survival curves were calculated according to the Kaplan-Meier method with the log-rank test. All reported $P$ values were two-sided, and $P$ less than 0.05 was considered statistically significant. SPSS 13.0 (SPSS Inc., Chicago, IL) was used for the statistical analysis.

\section{Results}

Among the 103 patients included in the study, the median age was 61 years (range, 36 to 79 years), and $61.2 \%$ (63 cases) were female. $41.7 \%$ (43 cases) of these patients had smoking history. The majority of tumors $(52.4 \%)$ are located in the upper lobe. With regard to histological grade in the 103 patients, $85.4 \%$ (88 cases) were well-moderate differentiated grade and $14.6 \%$ (15 cases) were poorly differentiated. According to the UICC/AJCC 7th TNM staging system, 63 (61.2\%) patients were stages I and II and $40(38.8 \%)$ patients were stage III. Majority of these patients $(77.7 \%)$ received adjuvant therapy after pulmonary resection. Based on the threshold value of our hospital, patients were divided into two groups according to pretreatment CEA value $(\geq 5.2 \mathrm{ng} / \mathrm{mL}$ and $<5.2 \mathrm{ng} / \mathrm{mL})$ or hemoglobin level $(\geq 115 \mathrm{~g} / \mathrm{L}$ and $<115 \mathrm{~g} / \mathrm{L})$. According to the results of receiver operating characteristic (ROC) curve analysis, the enrolled patients were also divided into two groups by the median value of PLR $(\geq 120$ and $<120)$. The summary of patients' characteristics are shown in Table 1. For the whole study population, the median follow-up from the time of surgery was 30 months. At the end of follow-up, 10 patients $(9.7 \%)$ developed local and regional recurrence. Metastasis to the brain, bone, liver, and lung occurred in 12 (11.6\%), 6 (5.8\%), $6(5.8 \%)$, and $3(2.9 \%)$ patients, respectively. The median number of the brain metastasis lesions was 3 (range, 1-16). The median time of the brain metastasis development was 12 months (range, 4-22 months).

Several clinical and pathological factors were found to be associated with the brain metastasis of resected lung adenocarcinoma patients on both univariate and multivariate analyses. In univariate analysis, $\mathrm{N} 2$ stage $(P=0.013)$, stage III $(P=0.016)$, increased CEA level $(P=0.006)$, and higher PLR value $(P=0.020)$ before treatment were associated with an increased risk of developing brain metastasis. Using all of these four high risk factors, we constructed a multivariate 
TABLE 1: Clinicopathologic characteristics of 103 lung adenocarcinoma patients.

\begin{tabular}{|c|c|c|}
\hline Characteristics & Number of patients & $\%$ \\
\hline \multicolumn{3}{|l|}{ Age, years } \\
\hline$\geq 60$ & 58 & 56.3 \\
\hline$<60$ & 45 & 43.7 \\
\hline \multicolumn{3}{|l|}{ Sex } \\
\hline Male & 40 & 38.8 \\
\hline Female & 63 & 61.2 \\
\hline \multicolumn{3}{|l|}{ Smoking status } \\
\hline Never & 60 & 58.3 \\
\hline Ever & 43 & 41.7 \\
\hline \multicolumn{3}{|l|}{ Tumor location } \\
\hline Upper lobe & 53 & 52.4 \\
\hline Nonupper lobe & 60 & 47.6 \\
\hline \multicolumn{3}{|l|}{ Histology grade } \\
\hline Well-moderate & 88 & 85.4 \\
\hline Poor & 15 & 14.6 \\
\hline \multicolumn{3}{|l|}{ T stage } \\
\hline T1-2 & 86 & 83.5 \\
\hline $\mathrm{T} 3-4$ & 17 & 16.5 \\
\hline \multicolumn{3}{|l|}{ N stage } \\
\hline N0-1 & 75 & 72.8 \\
\hline $\mathrm{N} 2$ & 28 & 27.2 \\
\hline \multicolumn{3}{|l|}{ TNM stage } \\
\hline I-II & 63 & 61.2 \\
\hline III & 40 & 38.8 \\
\hline \multicolumn{3}{|l|}{ Adjuvant therapy } \\
\hline Yes & 80 & 77.7 \\
\hline No & 23 & 22.3 \\
\hline \multicolumn{3}{|l|}{ CEA } \\
\hline$\geq 5.2 \mathrm{ng} / \mathrm{mL}$ & 36 & 40.0 \\
\hline$<5.2 \mathrm{ng} / \mathrm{mL}$ & 67 & 60.0 \\
\hline \multicolumn{3}{|l|}{$\mathrm{Hb}$} \\
\hline$\geq 115 \mathrm{~g} / \mathrm{L}$ & 92 & 89.3 \\
\hline$<115 \mathrm{~g} / \mathrm{L}$ & 11 & 10.7 \\
\hline \multicolumn{3}{|l|}{ PLR } \\
\hline$\geq 120$ & 51 & 49.5 \\
\hline$<120$ & 52 & 50.5 \\
\hline
\end{tabular}

CEA, carcinoembryonic antigen; Hb, hemoglobin; PLR, platelet to lymphocyte ratio.

Cox proportional hazards regression model. In multivariate model analysis, CEA above $5.2 \mathrm{ng} / \mathrm{mL}(P=0.014)$ and PLR $\geq$ $120(P=0.036)$ remained as the risk factors for brain metastasis (Table 2). In addition, the relationship between increased CEA level and tumor, patient, or metastatic characteristics was assessed. The results demonstrated that increased CEA level was associated with smoke history $(P<0.001)$, poorly differentiated histology grade $(P=0.005)$, and higher TNM stage $(P=0.011)$.

A new cut-off value, $15.6 \mathrm{ng} / \mathrm{mL}$, was determined by ROC analysis for CEA. The ROC curves were generated for increased CEA $(\geq 15.6 \mathrm{ng} / \mathrm{mL})$ or higher PLR $(\geq 120)$ and the

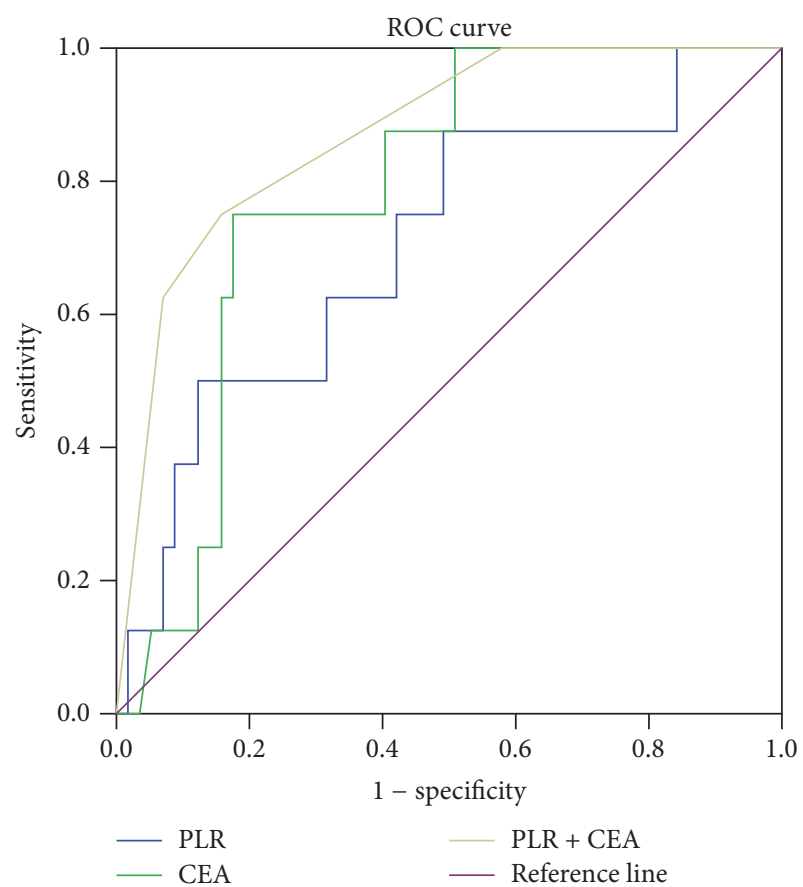

FIGURE 1: Receiving operator characteristic curve based on the sensitivity and specificity of CEA alone, PLR alone, or CEA and PLR combined.

combined analysis of these two factors. The combination of these two factors was superior to CEA alone or PLR alone in predicting brain metastasis according to the ROC analysis (AUC 0.872 versus 0.784 versus 0.704; Figure 1). These results indicated the potential predictive value of combined analysis of CEA and PLR in brain metastasis development of radical resected lung adenocarcinoma patients. The brain metastasis free survival curves, which are stratified by CEA, PLR, and the combination of these two factors, are shown in Figure 2.

\section{Discussion}

The majority of postoperative recurrences of NSCLC are distant metastasis, especially for brain metastasis [3]. Despite the advances in the treatment modalities [29-31], brain metastasis remains a major cause of mortality in patients with NSCLC. PCI has been investigated as a strategy to reduce the risk of brain metastasis for lung cancer patients. Several trials have shown that PCI is effective in reducing brain metastasis for NSCLC patients; however, there is no survival advantage [7]. The effort to identify lung cancer patients with high risk for developing brain metastasis would be able to avoid excessive treatment for patients with less aggressive tumors and increase the benefits of PCI $[8,9]$. In addition, the identification of high risk patients for developing brain metastasis could also be used to establish an optimal followup strategy for detecting brain relapse. Until now, brain CT or MRI is not routinely used in the follow-up of postoperative NSCLC patients. However, more and more evidences suggest that early detection of brain metastasis by brain CT or MRI 
TABLE 2: Univariate and multivariate Cox regression analyses estimating the risk factors of brain metastases of resected lung adenocarcinoma patients.

\begin{tabular}{|c|c|c|c|c|c|c|c|}
\hline \multirow{2}{*}{\multicolumn{2}{|c|}{ Clinicopathological factors }} & \multicolumn{3}{|c|}{ Univariable analysis } & \multicolumn{3}{|c|}{ Multivariable analysis } \\
\hline & & \multirow{2}{*}{$\begin{array}{c}\text { Hazard ratio } \\
1.514\end{array}$} & \multirow{2}{*}{$\begin{array}{c}95 \% \text { CI } \\
0.456-5.029\end{array}$} & \multirow{2}{*}{$\begin{array}{c}P \text { value } \\
0.498\end{array}$} & \multirow[t]{2}{*}{ Hazard ratio } & \multirow[t]{2}{*}{$95 \%$ CI } & \multirow[t]{2}{*}{$P$ value } \\
\hline Age, years & $\geq 60$ versus $<60$ & & & & & & \\
\hline Gender & Male versus female & 0.616 & $0.199-1.910$ & 0.402 & & & \\
\hline Smoking status & Ever versus never & 1.230 & $0.333-4.542$ & 0.756 & & & \\
\hline Tumor location & Upper lobe versus nonupper lobe & 0.698 & $0.222-2.200$ & 0.539 & & & \\
\hline Histology grade & Well versus poor & 0.873 & $0.263-2.898$ & 0.824 & & & \\
\hline T stage & T3-4 versus T1-2 & 2.593 & $0.780-8.615$ & 0.120 & & & \\
\hline $\mathrm{N}$ stage & N2 versus N0-1 & 4.304 & $1.365-13.575$ & 0.013 & 1.374 & $0.283-6.669$ & 0.693 \\
\hline TNM stage & III versus I-II & 4.976 & $1.346-18.390$ & 0.016 & 2.640 & $0.440-15.829$ & 0.288 \\
\hline Adjuvant therapy & Yes versus no & 1.175 & $0.318-4.343$ & 0.808 & & & \\
\hline CEA & $<5.2 \mathrm{ng} / \mathrm{mL}$ versus $\geq 5.2 \mathrm{ng} / \mathrm{mL}$ & 0.162 & $0.004-0.598$ & 0.006 & 0.194 & $0.052-0.722$ & 0.014 \\
\hline $\mathrm{Hb}$ & $\geq 115 \mathrm{~g} / \mathrm{L}$ versus $<115 \mathrm{~g} / \mathrm{L}$ & 0.510 & $0.111-2.330$ & 0.358 & & & \\
\hline PLR & $\geq 120$ versus $<120$ & 6.085 & $1.333-27.285$ & 0.020 & 5.149 & $1.117-23.729$ & 0.036 \\
\hline
\end{tabular}

$\mathrm{CI}$, confidence interval; CEA, carcinoembryonic antigen; Hb, hemoglobin; PLR, platelet to lymphocyte ratio.

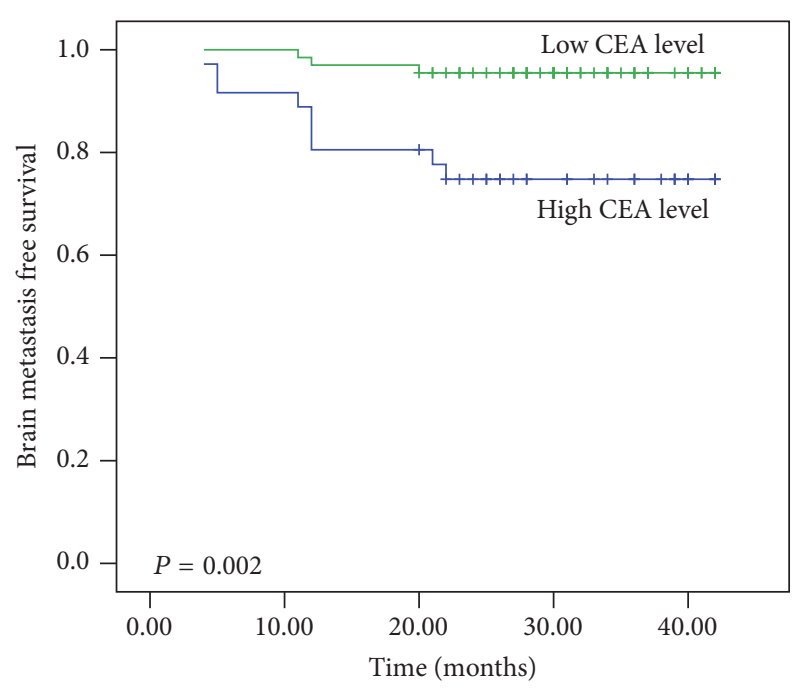

(a)

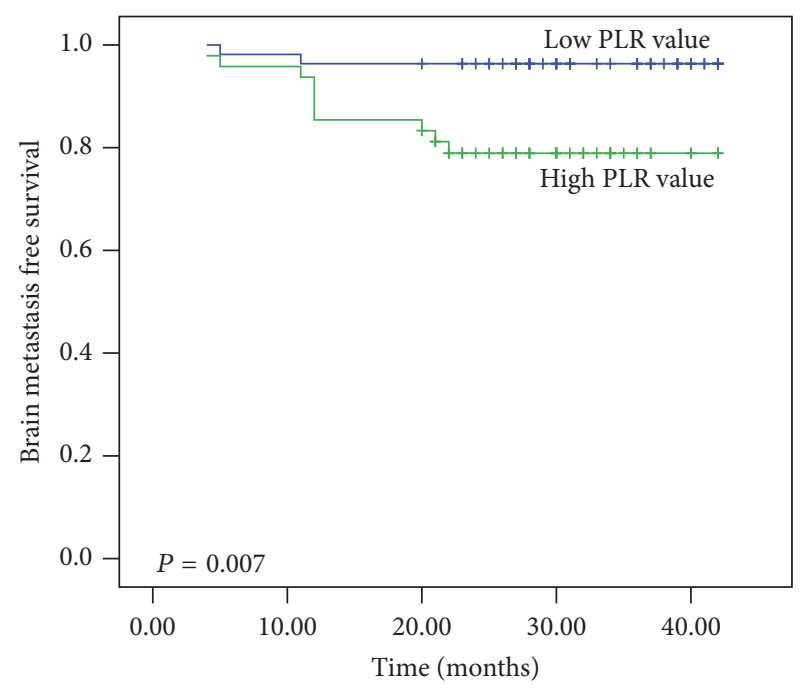

(b)

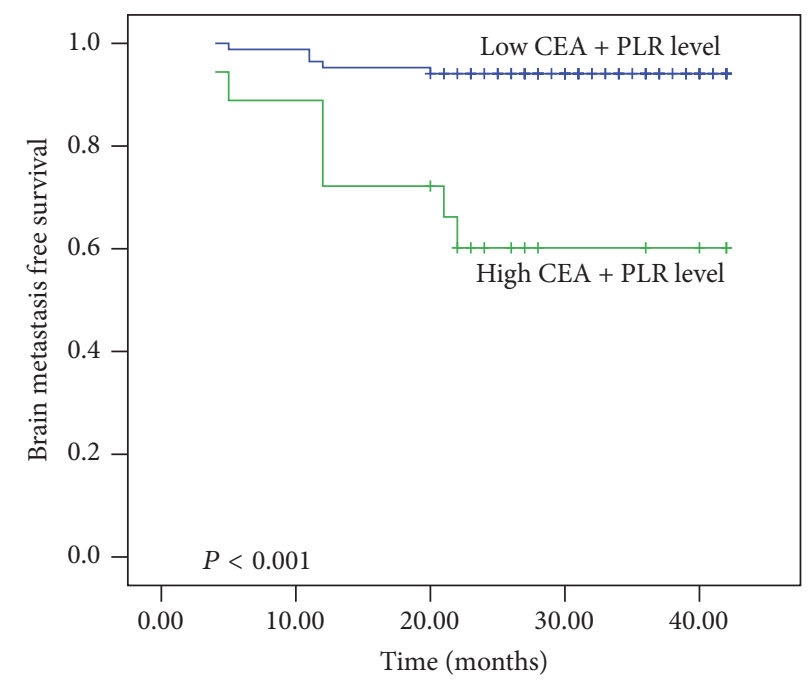

(c)

FIgURE 2: Brain metastasis free survival according to CEA alone (a), PLR alone (b), or CEA and PLR combined (c). 
during follow-up may improve the survival of lung cancer patients [32, 33]. As such, in this study, we focus on the identification of predictors for developing brain metastasis in resected lung adenocarcinoma patients, who are more likely to suffer brain metastasis.

In present study, $11.6 \%$ of the patients had developed brain metastases by 30 months of follow-up. In univariate analysis, N2 stage, stage III, increased CEA level, and higher PLR value were associated with an increased rate of brain metastasis. However, in multivariable analysis, only increased CEA level and higher PLR value were selected as the predictors for the probability of developing brain metastasis after curative surgery in lung adenocarcinoma patients.

CEA, a type of $\beta-1$ glycoprotein and a member of the IgCAM superfamily, is produced by the CEACAM5 gene and expressed during the early fetal life [11]. In the previous study, some groups had reported the association between pretreatment serum CEA level and the brain metastasis development of NSCLC. Lee et al. [15] found that the pretreatment serum CEA level was significantly correlated with brain metastasis in advanced NSCLC. The AUC of serum CEA for the prediction of brain metastasis was 0.724. Arrieta et al. [13] studied 293 patients with NSCLC in IIIB-IV clinical stage in a prospective manner. They indicated that high CEA serum level ( $\geq 40 \mathrm{ng} / \mathrm{mL}$ ) was a risk factor for brain metastasis development and was associated with poor prognosis in patients with advanced NSCLC. In current study, our findings also suggested that abnormal pretreatment serum CEA level was strongly correlated with increased brain metastatic potential in resected lung adenocarcinoma patients.

Inflammation is a hallmark of cancer [34]. More and more evidences show that the systemic inflammatory response is related to the initiation and progression of various forms of cancer $[16,17]$. PLR, the relative value of a combined platelet and lymphocyte counts, is a promising prognostic inflammation marker. Although the mechanisms underlying the association of PLR and prognosis of NSCLC are still incompletely understood, the relationship of PLR and the prognosis of NSCLC was explored in several studies [22, 2427]. One of the aims of the current study was to evaluate the value of the PLR in brain metastasis prediction. To our knowledge, this is the first study to investigate the association between the PLR and brain metastasis of NSCLC patients. The results of our study showed that higher pretreatment PLR value was a predictor of brain metastasis. Additionally, in the ROC analysis, the results demonstrated that AUC was 0.784 for CEA, 0.704 for PLR, and 0.872 for the combined analysis, indicating that the combination analysis was superior to CEA or PLR alone as a predictive factor in patients with lung adenocarcinoma who received complete resection.

As a retrospectively analysis, our study had some limitations. Firstly, the relatively small sample size may introduce the selection bias. Furthermore, due to the limited number of patients who had undergone EGFR status detection at the time of this study, the impact of EGFR status on the development of brain metastasis was not investigated in this study.

\section{Conclusion}

The present study demonstrates that increased CEA level and higher PLR value are independent risk factors for brain metastasis development of resected lung adenocarcinoma patients. Combined analysis CEA and PLR could improve the prediction efficacy of brain metastasis for completely resected lung adenocarcinoma patients. However, prospectively conducted studies are warranted to validate the results.

\section{Conflicts of Interest}

The authors declare that they have no conflicts of interest.

\section{Authors' Contributions}

Yan-Yang Wang, Jin-Xi He, and Ren Zhao conceived and designed the study. Wei Wang, Chao Bian, Di Xia, and Ping Hai performed the study. Wei Wang and Yan-Yang Wang analyzed the data. Yan-Yang Wang wrote the paper.

\section{Acknowledgments}

This study was supported by the Key Disciplines Supporting Program of General Hospital of Ningxia Medical University (2016) for the designing of the study and collection, analysis, and interpretation of data and writing of the manuscript.

\section{References}

[1] R. L. Siegel, K. D. Miller, and A. Jemal, "Cancer statistics, 2016," CA: A Cancer Journal for Clinicians, vol. 66, no. 1, pp. 7-30, 2016.

[2] K. D. Miller, R. L. Siegel, C. C. Lin et al., "Cancer treatment and survivorship statistics, 2016," CA Cancer Journal for Clinicians, vol. 66, no. 4, pp. 271-289, 2016.

[3] R. A. Figlin, S. Piantadosi, R. Feld et al., "Intracranial recurrence of carcinoma after complete surgical resection of Stage I, II, and III non-small-cell lung cancer," New England Journal of Medicine, vol. 318, no. 20, pp. 1300-1305, 1988.

[4] P. E. Van Schil, J. M. Hendricks, L. Carp, and P. R. Lauwers, "Surgery for oligometastatic disease in non-small-cell lung cancer," Expert Review of Anticancer Therapy, vol. 8, no. 12, pp. 1931-1938, 2008.

[5] A. Aupérin, R. Arriagada, J.-P. Pignon et al., "Prophylactic cranial irradiation for patients with small-cell lung cancer in complete remission," New England Journal of Medicine, vol. 341, no. 7, pp. 476-484, 1999.

[6] B. Slotman, C. Faivre-Finn, G. Kramer et al., "Prophylactic cranial irradiation in extensive small-cell lung cancer," New England Journal of Medicine, vol. 357, no. 7, pp. 664-672, 2007.

[7] C. L. Péchoux, A. Sun, B. J. Slotman, D. De Ruysscher, J. Belderbos, and E. M. Gore, "Prophylactic cranial irradiation for patients with lung cancer," The Lancet Oncology, vol. 17, no. 7, pp. e277-e293, 2016.

[8] F. Zhang, W. Zheng, L. Ying et al., "A Nomogram to Predict Brain Metastases of Resected Non-Small Cell Lung Cancer Patients," Annals of Surgical Oncology, vol. 23, no. 9, pp. 30333039, 2016.

[9] Y.-W. Won, J. Joo, T. Yun et al., "A nomogram to predict brain metastasis as the first relapse in curatively resected non-small 
cell lung cancer patients," Lung Cancer, vol. 88, no. 2, pp. 201207, 2015.

[10] H. J. Mamon, B. Y. Yeap, P. A. Jänne et al., "High risk of brain metastases in surgically staged IIIA non-small-cell lung cancer patients treated with surgery, chemotherapy, and radiation," Journal of Clinical Oncology, vol. 23, no. 7, pp. 1530-1537, 2005.

[11] N. Kokkonen, I. F. Ulibarri, A. Kauppila et al., "Hypoxia upregulates carcinoembryonic antigen expression in cancer cells," International Journal of Cancer, vol. 121, no. 11, pp. 24432450, 2007.

[12] D. Moro, D. Villemain, J. P. Vuillez, C. Agnius Delord, and C. Brambilla, "CEA, CYFRA21-1 and SCC in non-small cell lung cancer," Lung Cancer, vol. 13, no. 2, pp. 169-176, 1995.

[13] O. Arrieta, D. Saavedra-Perez, R. Kuri et al., "Brain metastasis development and poor survival associated with carcinoembryonic antigen (CEA) level in advanced non-small cell lung cancer: A prospective analysis," BMC Cancer, vol. 9, article no. 119, 2009.

[14] D. S. Lee, S. J. Kim, J. H. Kang et al., "Serum Carcinoembryonic antigen levels and the risk of whole-body metastatic potential in advanced nonsmall cell lung cancer," Journal of Cancer, vol. 5, no. 8, pp. 663-669, 2014.

[15] D.-S. Lee, Y.-S. Kim, S.-L. Jung et al., "The relevance of serum carcinoembryonic antigen as an indicator of brain metastasis detection in advanced non-small cell lung cancer.," Tumour biology: the journal of the International Society for Oncodevelopmental Biology and Medicine, vol. 33, no. 4, pp. 1065-1073, 2012.

[16] S. I. Grivennikov, F. R. Greten, and M. Karin, "Immunity, Inflammation, and Cancer," Cell, vol. 140, no. 6, pp. 883-899, 2010.

[17] F. Balkwill and A. Mantovani, "Inflammation and cancer: back to Virchow?” The Lancet, vol. 357, no. 9255, pp. 539-545, 2001.

[18] B. Azab, N. Shah, J. Radbel et al., "Pretreatment neutrophil/lymphocyte ratio is superior to platelet/lymphocyte ratio as a predictor of long-term mortality in breast cancer patients," Medical Oncology, vol. 30, no. 1, article no. 432, 2013.

[19] S. Raungkaewmanee, S. Tangjitgamol, S. Manusirivithaya, S. Srijaipracharoen, and T. Thavaramara, "Platelet to lymphocyte ratio as a prognostic factor for epithelial ovarian cancer," Journal of Gynecologic Oncology, vol. 23, no. 4, pp. 265-273, 2012.

[20] R. A. Smith, L. Bosonnet, M. Raraty et al., "Preoperative platelet-lymphocyte ratio is an independent significant prognostic marker in resected pancreatic ductal adenocarcinoma," American Journal of Surgery, vol. 197, no. 4, pp. 466-472, 2009.

[21] H.-C. Kwon, S. H. Kim, S. Y. Oh et al., "Clinical significance of preoperative neutrophil-lymphocyte versus plateletlymphocyte ratio in patients with operable colorectal cancer," Biomarkers, vol. 17, no. 3, pp. 216-222, 2012.

[22] S.-H. Kim, H. W. Lee, S.-I. Go, S. I. Lee, and G.-W. Lee, "Clinical significance of the preoperative platelet count and platelet-tolymphocyte ratio (PLT-PLR) in patients with surgically resected non-small cell lung cancer," Oncotarget, vol. 7, no. 24, pp. 3619836206, 2016.

[23] H. Zhang, H. Xia, L. Zhang, B. Zhang, D. Yue, and C. Wang, "Clinical significance of preoperative neutrophil-lymphocyte vs platelet-lymphocyte ratio in primary operable patients with non-small cell lung cancer," American Journal of Surgery, vol. 210, no. 3, pp. 526-535, 2015.

[24] Q.-T. Zhao, Z. Yuan, H. Zhang et al., "Prognostic role of platelet to lymphocyte ratio in non-small cell lung cancers: A meta-analysis including 3,720 patients," International Journal of Cancer, vol. 139, no. 1, pp. 164-170, 2016.
[25] H. Liu, Y. Wu, Z. Wang, Y. Yao, F. Chen, H. Zhang et al., "Pretreatment platelet-to-lymphocyte ratio (PLR) as a predictor of response to first-line platinum-based chemotherapy and prognosis for patients with non-small cell lung cancer," Journal of Thoracic Disease, vol. 5, pp. 783-789, 2013.

[26] G. Qiang, C. Liang, F. Xiao et al., "Prognostic significance of platelet-to-lymphocyte ratio in non-small-cell lung cancer: A meta-analysis," OncoTargets and Therapy, vol. 9, pp. 869-876, 2016.

[27] X. Gu, S. Sun, X.-S. Gao et al., "Prognostic value of platelet to lymphocyte ratio in non-small cell lung cancer: Evidence from 3,430 patients," Scientific Reports, vol. 6, Article ID 23893, 2016.

[28] K. Chansky, J.-P. Sculier, J. J. Crowley, D. Giroux, J. V. Meerbeeck, and P. Goldstraw, "The international association for the study of lung cancer staging project: Prognostic factors and pathologic TNM stage in surgically managed non-small cell lung cancer," Journal of Thoracic Oncology, vol. 4, no. 7, pp. 792801, 2009.

[29] N. M.-a. C. Group, R. Arriagada, A. Auperin, S. Burdett, J. P. Higgins, D. H. Johnson et al., "Adjuvant chemotherapy, with or without postoperative radiotherapy, in operable non-smallcell lung cancer: two meta-analyses of individual patient data," Lancet, vol. 375, pp. 1267-1277, 2010.

[30] A. Aupérin, C. Le Péchoux, E. Rolland et al., "Meta-analysis of concomitant versus sequential radiochemotherapy in locally advanced non - small-cell lung cancer," Journal of Clinical Oncology, vol. 28, no. 13, pp. 2181-2190, 2010.

[31] K. L. Johung, N. Yeh, N. B. Desai et al., "Extended survival and prognostic factors for patients with ALK-rearranged nonsmall-cell lung cancer and brain metastasis," Journal of Clinical Oncology, vol. 34, no. 2, pp. 123-129, 2016.

[32] K. Yokoi, N. Miyazawa, and T. Arai, "Brain metastasis in resected lung cancer: Value of intensive follow-up with computed tomography," Annals of Thoracic Surgery, vol. 61, no. 2, pp. 546-551, 1996.

[33] T. Nishikawa, T. Ueba, M. Kawashima et al., "Early detection of metachronous brain metastases by biannual brain MRI followup may provide patients with non-small cell lung cancer with more opportunities to have radiosurgery," Clinical Neurology and Neurosurgery, vol. 112, no. 9, pp. 770-774, 2010.

[34] D. Hanahan and R. A. Weinberg, "Hallmarks of cancer: the next generation," Cell, vol. 144, no. 5, pp. 646-674, 2011. 


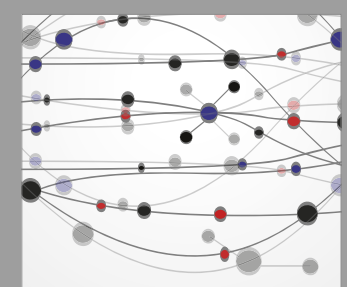

The Scientific World Journal
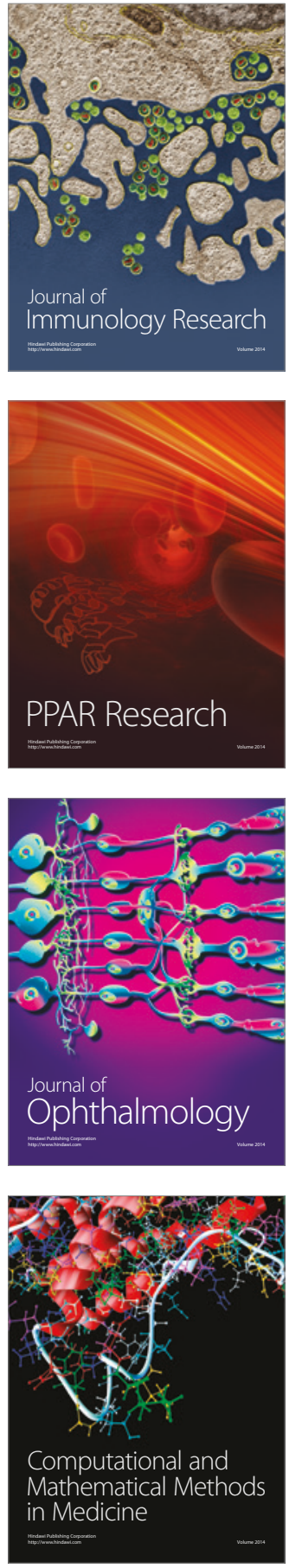

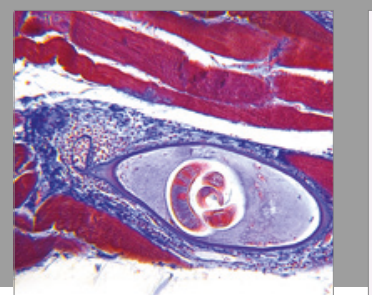

Gastroenterology Research and Practice
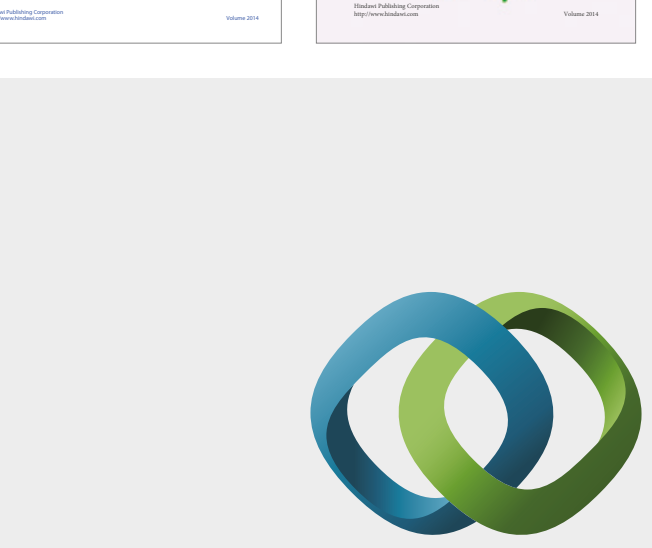

\section{Hindawi}

Submit your manuscripts at

https://www.hindawi.com
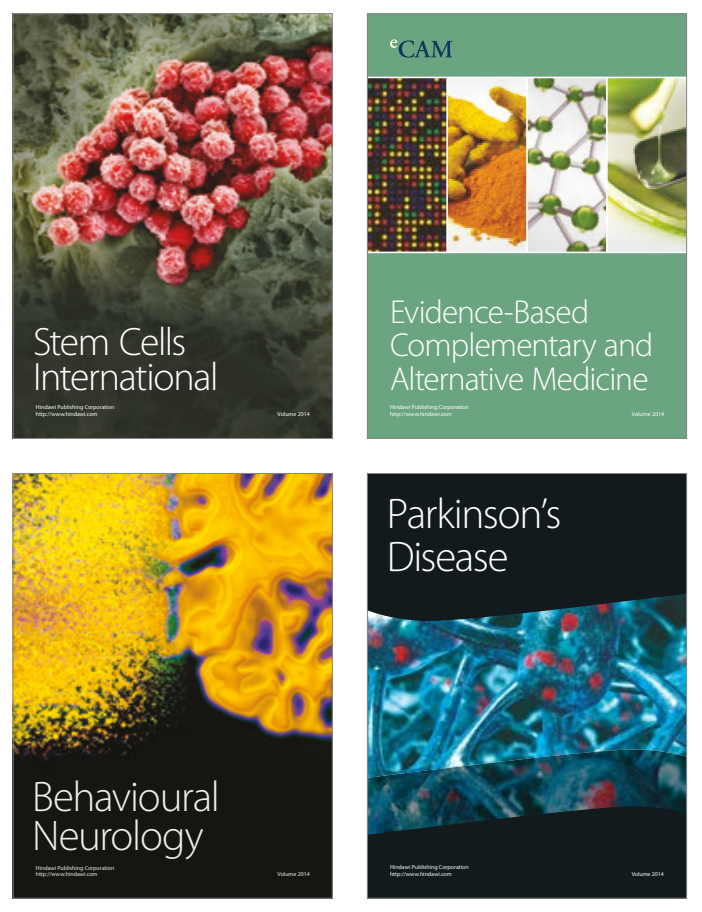
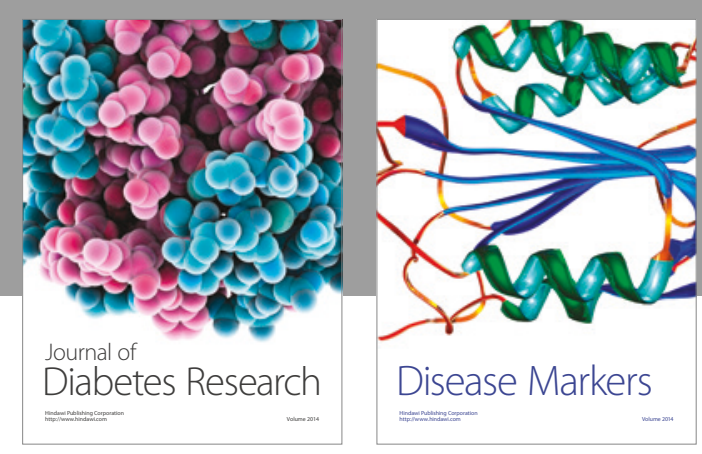

Disease Markers
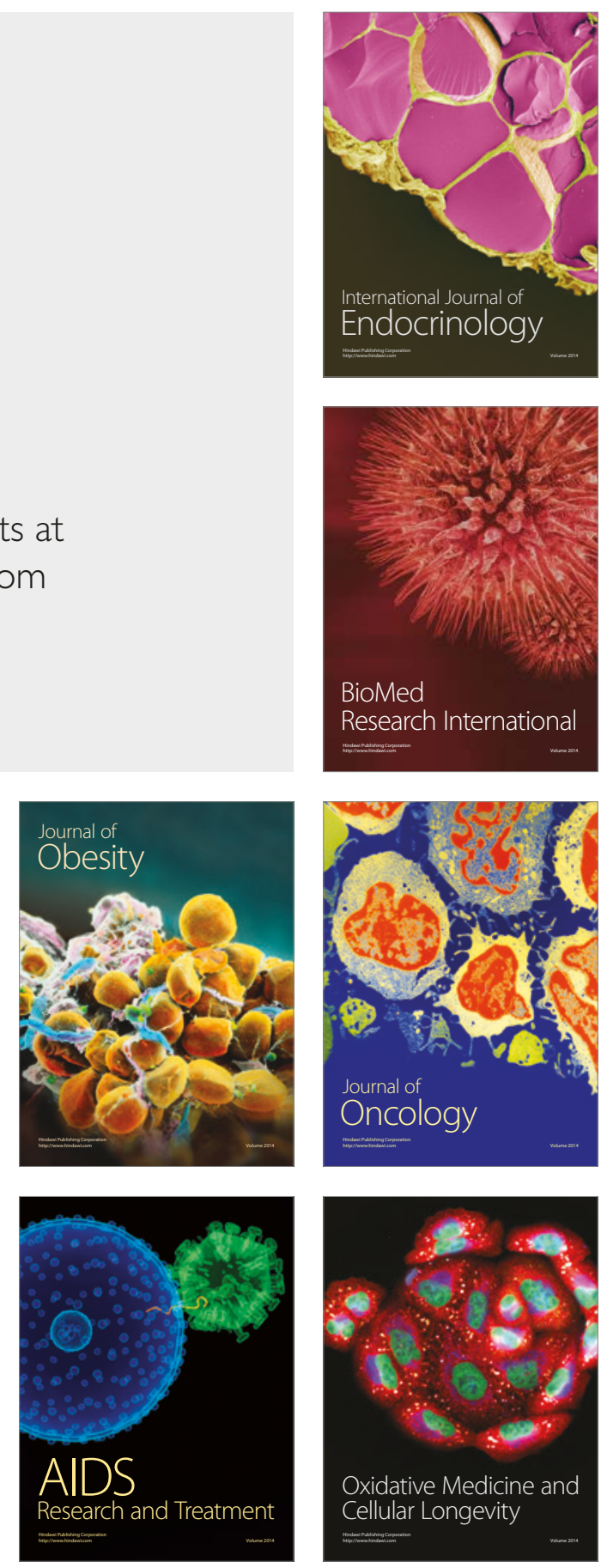